\title{
Non invasive delivery system for glucagon like peptide - I (glp-I): site specific guanidinylation and biotinylation of lysine residues in glp-I for treatment of type ii diabetes
}

\section{Introduction}

With the age there is a progressive impairment in the islets of beta cells of langerhans in pancreas. As a result a considerable fraction of population over the age of 65 is diagnosed with type II diabetes mellitus.

\section{Diabetes Mellitus type II}

Formerly non-insulin dependent diabetes mellitus (NIDDM) or adult onset diabetes is a metabolic disorder that is characterized by high blood glucose in the context of insulin and relative insulin deficiency. ${ }^{1-3}$ The condition is often initially managed by increasing exercise and dietary modification. However, if the condition progresses medication may be required. The condition is due to a combination of lifestyle and genetic factors. Obesity has been found to contribute to majority of cases of type II diabetes. The classic symptoms of diabetes are polyuria, polydipsia, polyphagia, fatigue and weight loss. Type II diabetes is a result of combination of lifestyle and genetic factors. The natural progression of type II diabetes necessitates most diabetes patients eventually to start insulin therapy. Insulin is a plays role in the control of lipid and carbohydrate metabolism. When glucose is released from dietary carbohydrates and absorbed, elevated concentrations of glucose in the blood stimulates the release of insulin. Insulin facilitates entry of glucose into adipose tissues, muscle and several other tissues. Insulin also stimulates the liver for storage of glucose in the form of glycogen. As blood glucose concentrations fall, insulin secretion stops. In the absence of insulin, the cells in the body will switch to using alternative fuels like fatty acids for energy, and subsequently, enzymes will break down the glycogen in the liver. Glucagon is the hormone which plays a major role for conversion of glycogen to glucose in liver which is then released into the blood stream.

\section{Lifestyle}

A number of lifestyle factors are known to be important for the development of diabetes type II. In a study, individuals with high levels of physical activity, taking healthy diet, did not smoke and were moderate alcohol consumer had $82 \%$ lower rate of diabetes as compared to normal weight individual, having the rate $89 \%$ lower. A healthy diet refers to one high in fiber, with a high polyunsaturated to saturated fat ratio and a lower mean glycemic index. Obesity has been found to be a major contributor approximately $55 \%$ of cases of type II diabetes. Decreasing consumption of saturated and trans fatty acids while replacing them with unsaturated fats may help in decreasing the risk of contracting the disease. ${ }^{2}$

\section{Genetic factors}

Type II diabetes has a strong inheritable genetic connection especially having first degree relatives with the disease increases the
Volume I Issue 4 - 2015

\author{
Saurabh Patel \\ Zifam Pinnacle Health Care, India
}

Correspondence: Saurabh Patel, Zifam Pinnacle Health Care, India,Tel+917043745065, Email patelsaul I@gmail.com

Received: May 23, 2015 | Published: September 19, 2015

risk of developing it. Presence of same trait in both members of a pair of monozygotic twins is close to $100 \%$ and about $25 \%$ of those having disease have a family history of diabetes. Also the mutational events (mutation of the Islet Amyloid polypeptide gene) results in early onset of more severe form of diabetes. ${ }^{2,4,5}$ Genes significantly associated with developing type II diabetes include FTO, NOTCH2, CDKAL1, SLC30A8, JAZF1, TCF7L2, HHEX, PPARG, KCNJ11, WFS1.

\section{Pathophysiology}

Insulin resistance means that the body cells are not able to respond or if at all they respond, inappropriately in presence of insulin. This is more complex than type 1 diabetes but easier to treat especially when detected early when insulin is still being produced. If improperly managed type II diabetes may lead to complications like renal failure, blindness, erectile dysfunction, arterial disease (like coronary artery disease), slow capacity to heal (most common in case of gangrene) etc. $^{2}$

\section{Glucagon-like-peptide- I}

Glucagon-like-peptide-1 is a hormone, encoded in the proglucagon gene. It is produced in the enteroendocrine L cells of the gut. The hormone is secreted in blood stream when food rich in fat, protein hydrolysate and/or glucose enter into the duodenal part of intestine. Its effect on the secretion of insulin and glucagon has drawn attention for research activity over past two decades resulting in use of exendin-4, naturally occurring GLP-1 receptor agonist for treatment of type II diabetes. GLP-1 acts upon a specific G-protein coupled receptor which is present in tissues other than the pancreas (major blood vessels, lungs, brain, heart, and kidney). The most widely studied cell activated by GLP-1 is beta cells of islets of langerhans in pancreas where its defining action is augmentation of glucose-induced insulin secretion., ${ }^{3,6}$ GLP-1 has a number of other functionally important effects that are relevant for treating type II diabetes: stimulation of all steps of insulin biosynthesis, restoration of glucose sensitivity to the islets, and stimulation of increased expression of the glucose transporter GLUT-2 and glucokinase. GLP-1 also has tropic effects on beta-cells: stimulation of beta-cell proliferation, enhancement 
of the differentiation of new beta-cells from progenitor cells in the pancreatic duct epithelium, and inhibition of both cytokine and fatty acid-induced apoptosis in beta-cells. GLP-1 inhibits glucagon secretion, which then leads to reduced hepatic glucose output.?

\section{Hindrances in delivery of GLP- I}

Although there have been many advances in peptide and protein drug delivery technologies, the administration of peptides and proteins are still dependent on the invasive methods which leads to poor patient compliance due to pain and discomfort associated with frequent dosing, required in the therapy. Thus a non invasive administration of anti-diabetic agents which can bypass the discomfort zone of patient is a major need. Among the non-invasive routes of drug delivery, oral route has always been the most preferred due to its maximum convenience. However, anti-diabetic peptides like GLP-1 are not available for oral administration owing to the following main hindrances in oral administration of peptides/GLP-1:

Proteolysis by gastrointestinal peptidases

Less permeation through the intestinal membrane due to their large molecular size and hydrophilic nature along with the tight building nature of the membrane.

Thus a delivery system which bypasses the above two barriers can be a potential oral delivery system for peptides like GLP- $1 .{ }^{8}$

\section{Proposed approach}

The site specific chemical modification of the GLP-1 molecule, such that, it not only facilitates its uptake by intestinal cells but also protects the peptide against enzymatic degradation (trypsin) in intestine, followed by loading of such modified GLP-1 analogue in a hydrogel matrix which shows $\mathrm{pH}$ responsive swelling and thus release of the peptide at desired site. It has been reported that $\mathrm{pH}$ responsive polymeric hydrogels can be used as effective carriers for peptide drugs.

To overcome the enzymatic and membrane barrier for efficient delivery of GLP-1 it has been demonstrated in a recent research papers by Youn et al. ${ }^{8}$ that Lys ${ }^{26,34}$-biotin-GLP-1 has a 9 fold higher oral hypoglycemic efficacy than the unmodified native GLP-1(736). The Lys ${ }^{26,34}$-Biotinylation of the GLP-1(7-36) leads to increased permeability, enzyme resistance as well as preserves the bioactivity The permeability of the modified GLP-1(7-36) was found to be $25 \%$ as compared to $3 \%$ of unmodified GLP-1(7-36).

It has been reported in recent article that Guanidinylation may be potential way to fabricate cell penetrating peptide analogue ${ }^{9}$ Guanidinium group is a structural component of HIV (human immunodeficiency virus) tat protein, an 86 -residue protein that can enter cells when added to the medium of cells in culture. Many peptides derived from HIV tat protein have cell penetrating effects with minimum domain of 49-57 residues. HIV tat (49-57) is rich in arginine and highly basic and the guanidinium groups in HIV tat are principally responsible for its uptake into the cells. When all the non arginine residues in HIV tat (49-57) are replaced with arginine residue it gave transporters with superior rates of uptake. Recently, it was proposed that the water-soluble positively charged guanidinium head groups of the transporter form bidentate hydrogen bonds with the H-bond acceptor functionality on the cell surface, and the resultant ion pair complexes partition into the lipoidal layer and migrate across the membrane. ${ }^{10,11}$

\section{Hypothesis}

The site specific Biotinylation at either of the Lysine residue $\left(\right.$ Lys $^{26}$ or Lys ${ }^{34}$ ) of GLP-1(7-36) when combined with site specific Guanidinylation of the other remaining Lysine residue $\left(\mathrm{Lys}^{26}\right.$ or Lys $^{34}$ ) of GLP-1 (7-36) may have a synergistic effect on transcellular transportation of GLP-1 across the intestinal membrane as well as it may offer resistance to inhibitory activity of intestinal enzyme trypsin.

\section{Objectives}

The main objectives of this paper are to study the effects of modifications of GLP-1(7-36):

Site specific Biotinylation of the Lysine ${ }^{26}$ residue of GLP-1(7-36) and Guanidinylation of the Lysine ${ }^{34}$ residue of GLP-1(7-36)

Site specific Biotinylation of the Lysine ${ }^{34}$ residue of GLP-1(7-36) and Guanidinylation of the Lysine ${ }^{26}$ residue of GLP-1(7-36)

\section{On}

Permeability across Caco-2 cell monolayer

Stability against intestinal enzymes

Bioactivities and oral hypoglycemic efficacies of the modified GLP-1s

\section{Innovation}

The novel approach lies in the chemical modification by Guanidinylation of the non-biotinylated Lysine residues at either of 26/34 positions and then the incorporation of so formed modified GLP-1 analogue in a hydrogel matrix which exhibits $\mathrm{pH}$ responsive swelling may help in design of optimized oral delivery system for GLP-1.

Optimization of oral delivery system of GLP-1 refers to the increase in resistance to trypsin activity, enhance membrane permeability and preserve biological activity of GLP-1 and protection from gastric pepsin by incorporation in polymeric hydrogels.

\section{Enhancement of resistance to trypsin}

GLP-1(7-36) consists of only two Lysine residues at position 26/34. As reported in studies with biotin as trans-membrane carrier, ${ }^{8}$ in a similar manner if guanidinium group is used for site specific Guanidinylation, it may protect the lysine ${ }^{26}$ or lysine ${ }^{34}$ positions of GLP-1(7-36) from trypsin mediated cleavage of their respective carboxyl sides. Trypsin not only exerts its strongest degradation activity at carboxyl terminals of lysine residues but it is also present in intestine area in high concentrations.

\section{Preservation of biological activity}

Due to clear difference between $\mathrm{pK}$ of $\alpha$ and $\varepsilon$ amine groups both Lys-amines 26/34 will be preferred for biotin substitution over the $\mathrm{N}$-terminal $\left(\mathrm{His}^{7}\right)^{8}$ The reaction may also take place at $\mathrm{N}$-terminal amine group which is reported to be a crucial region for biological action of GLP- $1 .{ }^{8}$ A Lys-amines specific reaction may be used for guanidinylation in a similar manner as that of biotinylation of GLP- $1^{8}$ to favor site specific guanidinylation leaving the $\mathrm{N}$-terminal amines unmodified. On contrary, both the Lys ${ }^{26,34}$ are weakly involved in biological activity of GLP-1 hence making them suitable as modification sites. 


\section{Enhancement of membrane permeability}

Water soluble, positively charged guanidinium groups forms hydrogen bonds with the negatively charged, hydrogen bond acceptor functional group on cell membranes and resultant ion pair complex partitions into lipoidal membrane and traverses across the membrane $\mathrm{e}^{10}$ Guanidinylation at $\varepsilon$-amine side chain of lysine yields homo arginine ${ }^{12}$ When the $\varepsilon$-amine of Lysine side chain reacts with guanidinylating agent yielding homo arginine, the GLP-1 analogue so formed may exhibit increased cellular uptake which may be attributed to the formed guanidinium group of homo arginine. Also the so formed GLP-1(736) analogue will have some structural homology with Liraglutide (a GLP-1(7-37) analogue), wherein liraglutide has end sequence--$\mathrm{Arg}^{34}-\mathrm{Gly}^{35}-\mathrm{Arg}^{36}-\mathrm{Gly}^{37}$ while the modified $\mathrm{Lys}^{34}$-guanidinylatedGLP-1(7-36) as discussed here will have end sequence ---HomoArg ${ }^{34}$ $\mathrm{Gly}^{35}-\mathrm{Arg}^{36}$.

\section{Protection from gastric pepsin}

The incorporation of the biotinylated and guanidinylated GLP-1s in a $\mathrm{pH}$ responsive polymeric hydrogel matrix may protect them from harsh gastric conditions of $\mathrm{pH} 1.2$ due to presence of pepsin. ${ }^{13}$ The polymeric hydrogel shows $\mathrm{pH}$ responsive swelling, wherein swelling occurs only at higher $\mathrm{pH}$ range of 7.4 as encountered in intestinal region while the hydrogel remains in unswollen state in gastric $\mathrm{pH}$ environment and protects the peptide from gastric enzymes.

\section{Experiments to be performed and methodology 8,12}

\section{Preparation of biotinylated or guanidinylated GLP- Is followed by guanidinylation or biotinylation respectively, for 2 lysine residues respectively}

Trypsin is used to cleave the native GLP-1 at specific sites. Biotinylated GLP-1s can be prepared by treating Tryptic peptides containing lysine at c-terminal with Biotin-N-hydroxy succinimide in an environment of TEA (triethylamine) and DMSO (dimethyl sulfoxide). A portion (10mg) of GLP-1 is mixed with an equimolar amount of biotin- NHS in $2.5 \mathrm{ml}$ of a $0.3 \%$ triethylamine/dimethyl sulfoxide solution at room temperature for 60min (Figure 1).

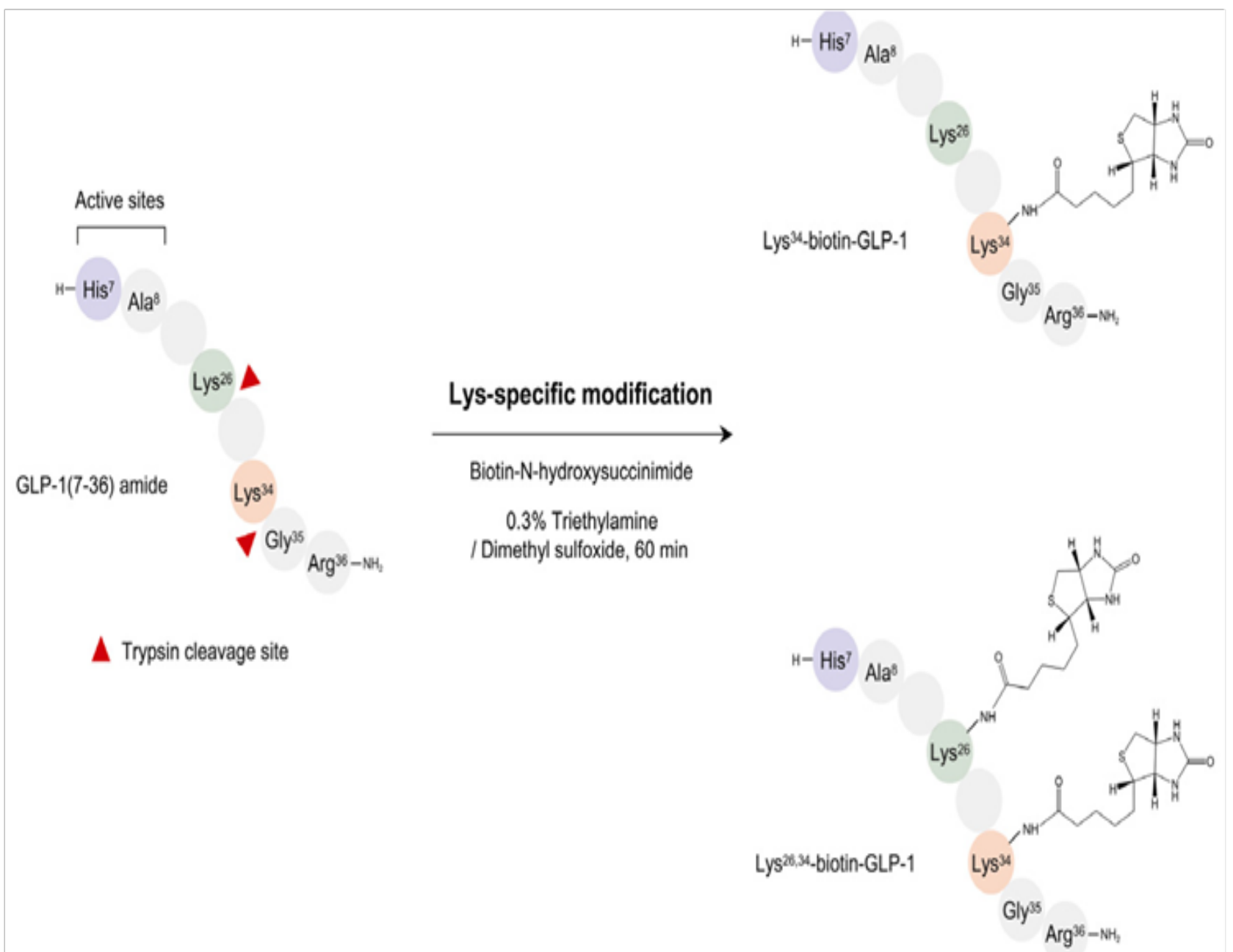

Figure I Tryptic peptides containing lysine at c-terminal with Biotin-N-hydroxy succinimide in an environment of TEA (triethylamine) and DMSO (dimethyl sulfoxide).

Guanidinylation may be performed prior to, or after biotinylation as favorable, with use of suitable protecting groups for other amine residues. The tryptic peptides of GLP-1 with lysine at $\mathrm{c}$ terminal (Lys- amine) when treated with $\mathrm{O}$-methylisourea (specific for $\varepsilon$-amine of lysine residue) give homoarginine ${ }^{12}$ (Figure 2). 


\section{Guanidination Reaction}
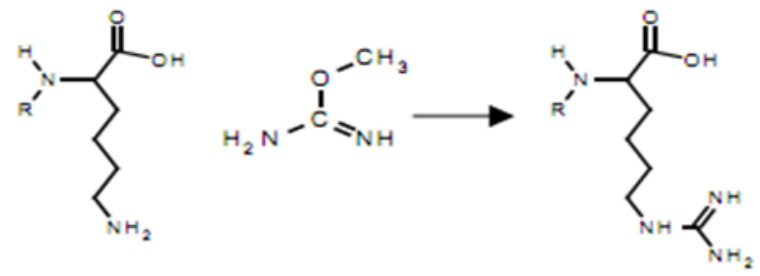

C-terminal lysine

Figure 2 Guanidination reaction.

\section{Characterization of modified GLP-I s}

Characterization of modified GLP-1s can be carried out by slight modification of MALDI-TOF MS method.

A $5 \mu 1$ aliquot of a lysyl endoproteinase Lys-C solution $(10 \mu \mathrm{g} / \mathrm{ml}$ in $50 \mathrm{mM}$ Tris- $\mathrm{HCl}, \mathrm{pH} 8.5$ ) will be added to $10 \mu 1$ of the modified GLP-1s $(100 \mu \mathrm{g} / \mathrm{ml})$ dissolved in the same buffer solution. Digestion should be allowed to continue at $37^{\circ} \mathrm{C}$ for $30 \mathrm{~min}$. Each sample will be subjected to MALDI-TOF Mass Spectrometry.

\section{Permeability studies across the Caco-2 cell monolayer ${ }^{8}$}

Permeability studies can be performed using human colon adenocarcinoma Caco-2 cells. Briefly, the cells can be grown until they reach $90 \%$ confluence and then will be seeded onto the collagencoated polycarbonated Transwell membrane inserts $(0.4 \mu \mathrm{m}$ pore size $1.13 \mathrm{~cm}^{2}$ area; Corning Costar, Cambridge, MA) The cell monolayer inserts should be used when the trans epithelial electrical resistance (TEER) values reaches $350-500 \mathrm{X} \cdot \mathrm{cm}^{2}$ (after a culture period of $18-21$ days) After washing the cell monolayer with the transport medium (pH 7.4), HBSS containing 10mM Hepes and $25 \mathrm{mM}$ glucose), $0.5 \mathrm{ml}$ of the transport medium containing the modified GLP-1s $(5 \mu \mathrm{M})$ and $1.5 \mathrm{ml}$ of the drug-free transport medium will be added to the apical and basolateral sides, respectively. In particular, peptide adsorption to the transwell plate can be prevented by exposing the basolateral side of the inserts to a bovine serum albumin solution $(10 \mathrm{mg} / \mathrm{ml})$ for $10 \mathrm{~min}$ followed by washing three times with the transport medium before the transport experiment. The inserts will be moved to the adjoining wells containing the same volume of fresh medium every $15 \mathrm{~min}$ for $1 \mathrm{~h}$. The concentration of the basolateral side solution can be determined using a commercially active GLP-1 RIA kit (Linco). The transport profiles of GLP-1s from basolateral to the apical side will be examined using the same procedure.

\section{Evaluation of intestinal enzyme stability of modified GLP-Is}

The proteolytic stabilities of the modified GLP-1s will be evaluated in the rat intestine fluid and homogenate. The intestine fluid and homogenate can be obtained from a $24 \mathrm{~h}$-pre-fasted SD rat. The intestine fluid can be collected by flushing the intestinal part (from the duodenum to end of the jejunum) with $5 \mathrm{ml}$ of $25 \mathrm{mM}$ phosphate buffer ( $\mathrm{pH}$ 6.4) and centrifugation at 12,000rpm for 10min. Followed by dilution of the supernatant 1:10 with the same buffer to slow the rapid breakdown of native GLP-1 by decreasing the enzyme activity. The homogenate can be prepared from the supernatant of the homogenized intestine segment of which the inner side has been rinsed five times again with a saline solution. A portion $(25 \mu l)$ of either a GLP-1 or modified GLP-1s solution $(200 \mu \mathrm{g} / \mathrm{ml}$ each) will be mixed with the same volume of the enzyme solutions (pre-incubated at $37^{\circ} \mathrm{C}$ for $15 \mathrm{~min}$ ), and incubated at $37^{\circ} \mathrm{C}$. At predetermined times the incubations should be stopped by adding $200 \mu 1$ of $1 \%$ TFA/DW and ice-cold methanol for the fluid and homogenate, respectively. The resulting mixtures will be centrifuged at $12,000 \mathrm{rpm}$ for $5 \mathrm{~min}$, and the residual amounts in supernatants will be analyzed by RP-HPLC.

\section{Evaluation of bioactivities of modified GLP-Is}

The biological activities of GLP-1s after modification will be assessed both in vitro and in vivo. In vitro activity can be evaluated using an isolated rat pancreatic islet. Male Sprague-Dawley (SD) rat pancreas can be inflated by injecting a cold Hank's balanced buffered salt solution (HBSS, pH 7.4) containing $1.5 \mathrm{mg} / \mathrm{ml}$ type $\mathrm{V}$ collagenase. The isolated islets can be purified by centrifugation with a stepwise Ficoll gradient and maintained with a RPMI 1640 culture medium supplemented with $10 \%$ fetal bovine serum and $1 \%$ penicillinstreptomycin at $37^{\circ} \mathrm{C}$. After 2 days, 20 islets will be incubated for $2 \mathrm{~h}$ in $2 \mathrm{ml}$ of Krebs-Ringer bicarbonate- HEPES in the same atmosphere at $37^{\circ} \mathrm{C}$. The insulin concentrations released after stimulating the modified GLP-1s can be determined using a rat insulin EIA kit $(\mathrm{n}=6$ each).

The in vivo activity can be evaluated using an oral glucose tolerance test (OGTT) in type 2 diabetic mice (7-8 weeks old). The mice (fasted for $18 \mathrm{~h}$ ), will receive an intraperitoneal injection of modified GLP-1s (10nmol $/ \mathrm{kg}$ ) 30min before oral administration of a $1.0 \mathrm{~g} / \mathrm{kg}$ dose of glucose $(\mathrm{n}=7 \mathrm{each})$. At predetermined times, the blood glucose levels will be determined using a one-touch blood glucose meter (ACCU-CHEK sensor). The total hypoglycemic degree (\%, vs. saline group) will be calculated as follows: $\left(\mathrm{AUC}_{\text {saline }}{ }_{0-180 \min }-\mathrm{AUC}_{\text {test }}\right.$, $\left.{ }_{0-180 \min }\right) / \mathrm{AUC}_{\text {saline, }} 0_{0-180 \mathrm{~min}} \mathrm{x} 100$. In both studies, saline and GLP-1 will be used as the control groups.

\section{Evaluation of oral hypoglycemic efficacies of modified GLP-Is}

The hypoglycemic efficacies of GLP-1 and modified GLP-1 in db/ $\mathrm{db}$ mice (7-8 weeks old) through the Peroral route can be assessed using an OGTT. The mice fasted for $18 \mathrm{~h}$ will receive consecutive oral administrations of $0.1 \mathrm{ml}$ of a $\mathrm{NaHCO}_{3}$ solution $(3 \%)$, as a gastric neutralizer and a $0.1 \mathrm{ml}$ sample solution of GLP-1s (each $15 \mathrm{nmol} /$ mice) containing $50 \%$ propylene glycol at $5 \mathrm{~min}$ intervals. After $30 \mathrm{~min}, 1.0 \mathrm{~g} / \mathrm{kg}$ dose of glucose $(0.2 \mathrm{ml})$ will be again administered orally to each mouse.

Also the studies related to hydrogel swelling, release of modified peptide from the hydrogel matrix needs to be carried out in addition to above mentioned studies. ${ }^{13}$

\section{Acknowledgements}

None.

\section{Conflict of interest}

The author declares no conflict of interest.

\section{References}

1. Vinay Kumar, Nelson F, Abbas Abul K. Robbins and Cotran Pathologic Basis of Disease. 7th ed. USA: Saunders; 2005. p. 1194-1195.

2. Riserus U, Willett WC, Hu FB. Dietary fats and prevention of type 2 diabetes. Prog Lipid Res. 2009;48(1):44-51. 
3. Transepithelial Delivery of Peptides with Incretin Hormone Activities. Patent No. 7442682.

4. Sakagashira S, Sanke T, Hanabusa T, et al. Missense mutation of amylin gene (S20G) in Japanese NIDDM patients. Diabetes. 1996;45(9):1279_ 1281 .

5. Cho YM, Kim M, Park KS, et al. S20G mutation of the amylin gene is associated with a lower body mass index in Korean type 2 diabetic patients. Diabetes Res Clin Pract. 2003;60(2):125-129.

6. Doyle ME, Egan JM. Mechanisms of Action of GLP-1 in the Pancreas Pharmacol Ther. 2007;113(3):546-593.

7. Philippe J. The Role of GLP-1 in the Treatment of Type 2 Diabetes Rev Med Suisse. 2009;5(206):1260-1262, 1264-1265.

8. Youn YS, Chae SY, Lee S, et al. Improved peroral delivery of glucagonlike peptide-1 by site-specific biotin modification: Design, preparation and biological evaluation. Eur J Pharm Biopharm. 2008;68(3):667675 .
9. Zha XY, Sun P, Luo YF, et al. Guanidinylation: A simple way to fabricate cell penetrating peptide analogue-modified chitosan vector for enhanced gene delivery. Journal of Applied Polymer Sci. 2011;121(6):3569.

10. Rothbard JB, Jessop TC, Lewis RS, et al. Role of Membrane Potential and Hydrogen Bonding in the Mechanism of Translocation of Guanidinium-Rich Peptides into Cells. $J$ Am Chem Soc. 2004;126(31):9506-9507.

11. www.niper.co.in

12. https://www.sigmaaldrich.com/content/dam/sigma-aldrich/docs/ Sigma/Bulletin/ms0100bul.pdf

13. Nakamura K, Murray RJ, Joseph JI, et al. Oral insulin delivery using P (MAA-g-EG) hydrogels: effects of network morphology on insulin delivery characteristics. J Control Release. 2004;95(3):589-599. 